\title{
Application of Transesophogeal Echocardiography in Percutaneous Left Atrial Appendage Occlusion
}

\author{
Yin Huang', Ziqing Gao², Xiaobo Chen², Yongquan Huang, 2,* \\ ${ }^{1}$ Department of Cardiovascular, the Fifth Affiliated Hospital of Sun Yat-Sen University, China \\ ${ }^{2}$ Department of Ultrasound, the Fifth Affiliated Hospital of Sun Yat-Sen University, China
}

Received May 24, 2020; Revised July 24, 2020; Accepted September 11, 2020

\section{Cite This Paper in the following Citation Styles}

(a): [1] Yin Huang, Ziqing Gao, Xiaobo Chen, Yongquan Huang, "Application of Transesophogeal Echocardiography in Percutaneous Left Atrial Appendage Occlusion," International Journal of Cardiovascular and Cerebrovascular Disease, Vol. 7, No. 2, pp. 7 - 10, 2020. DOI: 10.13189/ijccd.2020.070201.

(b): Yin Huang, Ziqing Gao, Xiaobo Chen, Yongquan Huang (2020). Application of Transesophogeal Echocardiography in Percutaneous Left Atrial Appendage Occlusion. International Journal of Cardiovascular and Cerebrovascular Disease, 7(2), 7 - 10. DOI: 10.13189/ijccd.2020.070201.

Copyright $\mathrm{C} 2020$ by authors, all rights reserved. Authors agree that this article remains permanently open access under the terms of the Creative Commons Attribution License 4.0 International License

\begin{abstract}
Objective: To evaluate the use of transesophogeal echocardiography (TEE) for percutaneous left atrial appendage (LAA) occlusion. Methods: LAA occlusion was performed in 20 atrial fibrillation (AF) patients with non-valvular lesions, including nine males and 11 females. TEE was used for the preoperative measurement of the maximal LAA orifice diameter and LAA depth and the guidance of atrial septum puncture. The release of the occluder during the occlusion procedure was also performed under TEE monitoring. Results: All 20 patients underwent successful occlusion of the LAA under the guidance of TEE. There were two (10.0\%) cases with mild residual shunt after occlusion, among which only one $(5.0 \%)$ patient still retained slight residual shunt at one month postoperative. All the other $(95.0 \%)$ patients revealed no residual shunt. There was no difference between the maximal LAA orifice diameter $(22.75 \pm 4.85$ $\mathrm{mm}$ vs. $22.15 \pm 4.23)$ and LAA depth $(36.60 \pm 5.51$ vs. $35.00 \pm 4.76)$ derived from TEE and the digital subtraction angiography (DSA) measurement. Both the max orifice diameter and LAA depth measured by TEE were strongly correlated with that measured by DSA, with $\mathrm{r}=0.75, P<$ 0.001 and $\mathrm{r}=0.82, P<0.001$, respectively. Conclusions: TEE can accurately estimate the maximal LAA orifice diameter and LAA depth and provide an important reference for preoperative occluder size selection. It can also be used for intraoperative guidance and assessing results during the operation. TEE is of great importance for LAA occlusion.
\end{abstract}

Keywords Transesophogeal Echocardiography, Percutaneous Left Atrial Appendage Occlusion, Atrial Fibrillation

\section{Introduction}

Atrial fibrillation (AF), one of the most common arrhythmias, is a serious cardiac condition and a growing health problem [1]. From 2010 to 2030, the incidence of $\mathrm{AF}$ is expected to double [2]. However, AF post-diagnosis survival rates have not improved despite their steady increase in prevalence. The overall estimated five-year mortality after AF diagnosis is only $41 \%$ [3]. One reason for poor prognosis is AF often leads to thrombus formation. The left atrial appendage (LAA) is the most common site for thrombosis, accounting for $91 \%$ of left heart thrombi in patients with nonrheumatic AF [4], which is defined as rhythmic disturbance in the absence of rheumatic mitral stenosis, mechanical or bioprosthetic heart valve, or mitral valve repair [5].

Oral anticoagulants are the primary methods for preventing embolism. However, bleeding risk is a contraindication to anticoagulation [6]. Efforts have been made to develop techniques to reduce thromboembolic events in AF patients without the use of long-term oral anticoagulants. LAA occlusion is a minimally invasive treatment for preventing LAA thrombosis, and it is 
increasingly being used in $\mathrm{AF}$ patients who are at risk of bleeding and contraindicated in anticoagulation, have poor compliance with coagulation monitoring, or still have thrombus formation after anticoagulation [7].

Accurately measuring the orifice diameter and depth of the LAA before occlusion is crucial for the selection of an appropriate occluder. There are several imaging techniques for evaluating the size of the LAA, such as computerized tomography and magnetic resonance imaging. However, high costs and long examination times limit their clinical application. With the advantages of being real-time, simple, high resolution, and low cost, transesophageal echocardiography (TEE) is suitable for LAA examination. This study evaluated the use of TEE in patients with non-valvular lesions of $\mathrm{AF}$ who underwent LAA occlusion.

\section{Materials and Methods}

\subsection{Subjects}

We retrospectively reviewed 20 patients with AF who underwent TEE examinations for preoperative evaluation and intraoperative monitoring from January 2019 to April 2020. All patients gave written informed consent before examination.

\subsection{Inclusion Criteria and Exclusion Criteria}

Inclusion criteria: (1) persistent $\mathrm{AF}$ caused by nonrheumatic valvular disease, $\mathrm{AF}$ occurring $>3$ months; (2) age $>18$ years; (3) long-term use of clopidogrel and aspirin; (4) warfarin use contraindications.

Exclusion criteria: (1) thrombosis in the left atrium or other heart chambers; (2) the maximum orifice diameter of the LAA is smaller than $17 \mathrm{~mm}$ or larger than $31 \mathrm{~mm}$, or larger than the maximum depth of the LAA; (3) left ventricular systolic function is lower than $30 \%$; (4) atrial septal defect, or other congenital heart disease; (5) contraindications for TEE examination.

\subsection{Instruments and Methods}

A Philips IE Elite with X 2-7 transesophageal probe was employed to perform TEE examinations. Its scanning frequency is $2-7 \mathrm{MHz}$. The maximum orifice diameter and depth of the LAA was measured at multiple angles before operation.

\subsection{Statistical Analysis}

Continuous measurements were expressed as mean \pm standard deviation, and categorical measurements were expressed as frequencies and percentages. Comparisons between baseline data and follow-up data were performed using a paired $t$-test for continuous variables and
Pearson's chi-squared test or Fisher's exact test for categorical variables, as appropriate. The associations were tested by Spearman's correlation analysis. For all analyses, $P<0.05$ was considered significant. We performed all statistical comparisons using SPSS Statistics Version 13.0.

\section{Result}

\subsection{Baseline Information}

The subjects included nine males and 11 females, aged from 53 to $85(70.00 \pm 10.67)$ years old. TEE examinations were performed for both preoperative examination and intraoperative monitoring. Among these patients, three (15.0\%) had simple AF, and $17(85.0 \%)$ had other heart diseases. Nine (45.0\%) patients revealed an inner diameter of the left atrium more than $40 \mathrm{~mm}$. $\mathrm{N}$-terminal pro b-type natriuretic peptide was $\leq 300 \mathrm{pg} / \mathrm{ml}$ in four $(20.0 \%)$ cases, $300-1000 \mathrm{pg} / \mathrm{ml}$ in six $(30.0 \%)$ cases, and $>1000 \mathrm{pg} / \mathrm{ml}$ in $10(50.0 \%)$ cases.

\subsection{LAA Occlusion Results}

All 20 patients were successfully placed with an LAA Watchman occluder. There were two $(10.0 \%)$ cases with mild residual shunt after occlusion, and $18(90.0 \%)$ cases without residual shunt. Among the two patients with residual shunt after occlusion, only one $(5.0 \%)$ patient still retained slight residual shunt at one month postoperative in TEE follow-up, and the other (95.0\%) patients revealed no residual shunt.

\subsection{Comparison of the LAA Measurements between DSA and TEE}

Compared to the values measured by digital subtraction angiography (DSA), the maximum orifice diameter and depth of the LAA measured by TEE was not statistically different $(P>0.05$, Table 1$)$.

Table 1. Compare measurements of the orifice diameter and depth of the LAA between TEE and DSA

\begin{tabular}{|c|c|c|c|}
\hline & $\begin{array}{c}\text { Orifice diameter of } \\
\text { the LAA }\end{array}$ & $\begin{array}{c}\text { Depth of the } \\
\text { LAA }\end{array}$ & $P$ value \\
\hline TEE $(\mathrm{mm})$ & $22.75 \pm 4.85$ & $36.60 \pm 5.51$ & $>0.05$ \\
\hline DSA $(\mathrm{mm})$ & $22.15 \pm 4.23$ & $35.00 \pm 4.76$ & $>0.05$ \\
\hline
\end{tabular}

\subsection{Correlation between the LAA Measurements of DSA and TEE}

Both the max orifice diameter and depth of the LAA measured by TEE were strongly correlated with that measured by DSA, with $\mathrm{r}=0.75, P<0.001$ (Figure 1) and $\mathrm{r}=0.82, P<0.001$ (Figure 2), respectively. 


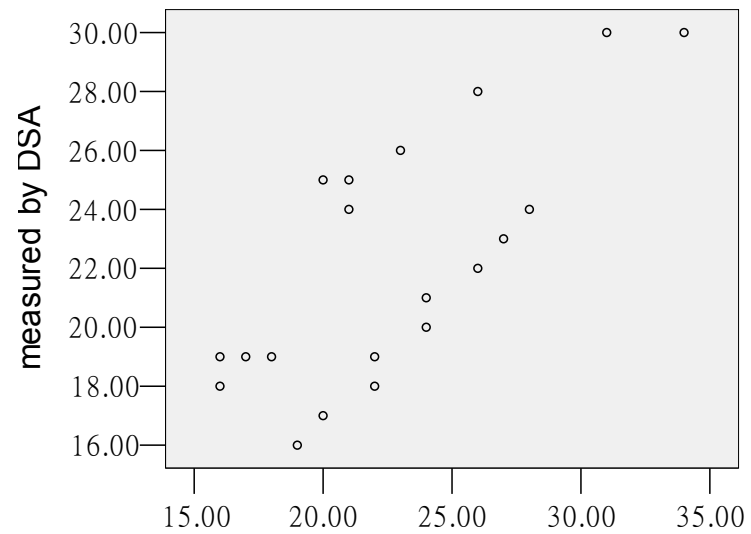

Figure 1. Corelationship between the orifice diameter of the LAA measured by TEE and DSA

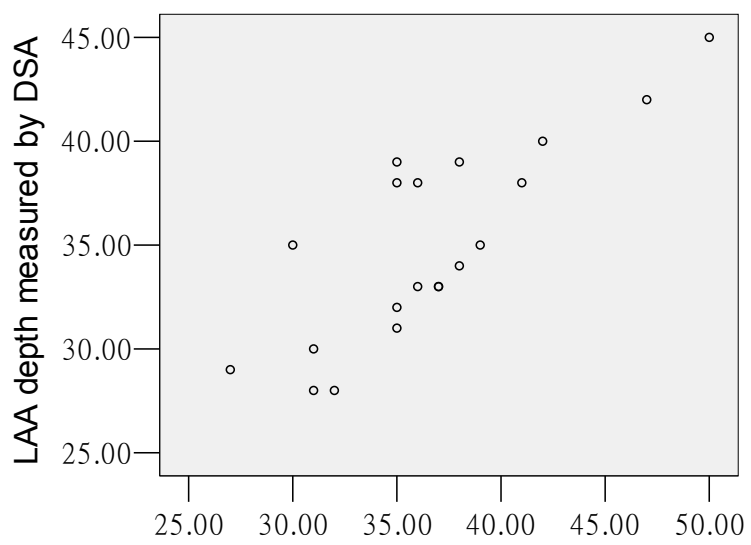

Figure 2. Corelationship between the depth of the LAA measured by TEE and DSA

\section{Discussion}

The endocardial surface of the LAA has a complex trabeculated structure with a variable degree of pectinate muscles [8]. The blood flows slowly like a vortex in the LAA of AF patients. This results in easy clot formation in crevices between pectinate muscles and causes serious complications such as cerebral infarction $[9,10]$. Evidence indicates that thrombosis in the LAA is the main cause of stroke in AF patients [11]. LAA occlusion is a feasible and increasingly used method for preventing thrombosis formation in patients with $\mathrm{AF}$ [12]. A multicenter clinical study revealed that LAA occlusion can reduce the risk of stroke, major bleeding, and death in AF patients compared to other strategies [13]. However, the anatomy of the LAA is complex and varies from patient to patient. Selecting a suitable occluder is essential for successful percutaneous LAA closure procedures. Undoubtedly, accurate measurement and shape details of the LAA are crucial for choosing an occluder.
For the guidance of LAA occlusion, TEE is currently the most used imaging modality [14]. Implanters routinely use TEE imaging to assess LAA device size selection, residual shunting, and final device placement [15]. Multiple plane TEE can show each side of the LAA clearly, and enable observation of the presence of a thrombus and measurement of the diameters and depth of the LAA [16]. This study focused on the application of TEE in patients with AF before and during occlusion of the LAA. We have found that there was no significant difference between the LAA orifice diameter, depth measured by TEE and their relative values measured by DSA. These data indicate that TEE assessment is suitable for screening candidates for device closure of the LAA. Additionally, by using biplane imaging in TEE during the occlusion procedure, the operator can puncture the atrial septum at a precise position and accurately send the occluder into the LAA. TEE guidance can improve trans-septal puncture safety and overall procedural success [17].

Moreover, we found the results of TEE examination showed a strong correlation with DSA measurements. TEE is convenient for operation and can provide clear structural information about the LAA. It can also help with clear monitoring during the pulling test of the occluder. Following the successful release of the plugging device, TEE can also evaluate its position and residual shunt at multiple angles and on multiple planes. More importantly, TEE can dynamically display the changes in the above observation indexes in real-time during the pushing and pulling experiments [16]. Also, TEE can further assess the surgical results conveniently and comprehensively. All of the above demonstrate that TEE can be used for determining whether there is thrombosis, and accurately measuring the diameters and clearly showing the shape of the LAA before interventional operation. It is certainly of great value for LAA occlusion.

However, in order to strengthen our conclusions, there are still some aspects worthy of attention. Firstly, this study was a retrospective static case analysis. The cases included in this study were somewhat limited, so large-scale prospective studies with more cases would help to validate our conclusion. Furthermore, TEE also plays an important role in postoperative examination. Therefore, long-term follow-up warrants further study.

\section{Conclusions}

TEE can accurately estimate the maximal LAA orifice diameter and LAA depth and provide an important reference for preoperative occluder size selection. It can also be used for intraoperative guidance and assessing results during operation. TEE is of great importance for LAA occlusion. 


\section{Acknowledgments}

The authors do not report any potential conflict of interest relevant to this article.

\section{REFERENCES}

[1] K. Subramaniam, A. Ibarra, M.L. Boisen, Echocardiographic Guidance of AMPLATZER Amulet Left Atrial Appendage Occlusion Device Placement, Semin Cardiothorac Vasc Anesth 23(2) (2019) 248-255.

[2] S. Colilla, A. Crow, W. Petkun, D.E. Singer, T. Simon, X. Liu. Estimates of current and future incidence and prevalence of atrial fibrillation in the U.S. adult population, Am J Cardiol 112(8) (2013) 1142-7.

[3] B.A. Williams, A.M. Honushefsky, P.B. Berger, Temporal Trends in the Incidence, Prevalence, and Survival of Patients with Atrial Fibrillation From 2004 to 2016, Am J Cardiol 120(11) (2017) 1961-1965.

[4] A.S. Go, E.M. Hylek, K.A. Phillips, Y. Chang, L.E. Henault, J.V. Selby, D.E. Singer, Prevalence of diagnosed atrial fibrillation in adults: national implications for rhythm management and stroke prevention: The AnTicoagulation and Risk Factors in Atrial Fibrillation (ATRIA) Study, JAMA 285(18) (2001) 2370-5.

[5] C.T. January, L.S. Wann, J.S. Alpert, H. Calkins, J.E. Cigarroa, J.C. Cleveland, Jr., J.B. Conti, P.T. Ellinor, M.D. Ezekowitz, M.E. Field, K.T. Murray, R.L. Sacco, W.G. Stevenson, P.J. Tchou, C.M. Tracy, C.W. Yancy, A.A.T.F. Members, 2014 AHA/ACC/HRS guideline for the management of patients with atrial fibrillation: executive summary: a report of the American College of Cardiology/American Heart Association Task Force on practice guidelines and the Heart Rhythm Society, Circulation 130(23) (2014) 2071-104.

[6] D. Altszuler, A.F. Vainrib, D.G. Bamira, R.J. Benenstein, A. Aizer, L.A. Chinitz, M. Saric, Left Atrial Occlusion Device Implantation: The Role of the Echocardiographer, Curr Cardiol Rep 21(7) (2019) 66.

[7] A.J. Camm, G.Y. Lip, R. De Caterina, I. Savelieva, D. Atar, S.H. Hohnloser, G. Hindricks, P. Kirchhof, E.S.C.C.f.P. Guidelines-CPG, R. Document, 2012 focused update of the ESC Guidelines for the management of atrial fibrillation: an update of the 2010 ESC Guidelines for the management of atrial fibrillation--developed with the special contribution of the European Heart Rhythm Association, Europace 14(10) (2012) 1385-413.
[8] G. Ernst, C. Stollberger, F. Abzieher, W. Veit-Dirscherl, E. Bonner, B. Bibus, B. Schneider, J. Slany, Morphology of the left atrial appendage, Anat Rec 242(4) (1995) 553-61.

[9] T. Saito, Y. Kawamura, Y. Tanabe, A. Asanome, K. Takahashi, J. Sawada, T. Katayama, N. Sato, H. Aizawa, N. Hasebe, Cerebral microbleeds and asymptomatic cerebral infarctions in patients with atrial fibrillation, J Stroke Cerebrovasc Dis 23(6) (2014) 1616-22.

[10] D.D. Wang, T.J. Forbes, J.C. Lee, M.H. Eng, Echocardiographic Imaging for Left Atrial Appendage Occlusion: Transesophageal Echocardiography and Intracardiac Echocardiographic Imaging, Interv Cardiol Clin 7(2) (2018) 219-228.

[11] V.Y. Reddy, D. Holmes, S.K. Doshi, P. Neuzil, S. Kar, Safety of percutaneous left atrial appendage closure: results from the Watchman Left Atrial Appendage System for Embolic Protection in Patients with AF (PROTECT AF) clinical trial and the Continued Access Registry, Circulation 123(4) (2011) 417-24.

[12] R.J. Bajwa, L. Kovell, J.R. Resar, A. Arbab-Zadeh, K. Mandal, H. Calkins, R.D. Berger, Left atrial appendage occlusion for stroke prevention in patients with atrial fibrillation, Clin Cardiol 40(10) (2017) 825-831.

[13] S. Panikker, J. Lord, J.W. Jarman, S. Armstrong, D.G. Jones, S. Haldar, C. Butcher, H. Khan, L. Mantziari, E. Nicol, W. Hussain, J.R. Clague, J.P. Foran, V. Markides, T. Wong, Outcomes and costs of left atrial appendage closure from randomized controlled trial and real-world experience relative to oral anticoagulation, Eur Heart J 37(46) (2016) 3470-3482.

[14] J. Saw, Intracardiac Echocardiography for Endovascular Left Atrial Appendage Closure: Is it Ready for Primetime?, JACC Cardiovasc Interv 10(21) (2017) 2207-2210.

[15] Y. Matsuo, P. Neuzil, J. Petru, M. Chovanec, M. Janotka, S. Choudry, J. Skoda, L. Sediva, M. Kurabayashi, V.Y. Reddy, Left Atrial Appendage Closure Under Intracardiac Echocardiographic Guidance: Feasibility and Comparison with Transesophageal Echocardiography, J Am Heart Assoc 5(10) (2016).

[16] J. Zhao, F. Li, Y. Zhang, Z. Zhuang, M. Wang, L. Fu, Y. Ni, Z. Lu, Z. Chen, C. Zhang, Left Atrial Appendage Occlusion Guided Only by Transesophageal Echocardiography, Cardiol Res Pract 2019 (2019) 1376515.

[17] G. Nucifora, F.F. Faletra, F. Regoli, E. Pasotti, G. Pedrazzini, T. Moccetti, A. Auricchio, Evaluation of the left atrial appendage with real-time 3-dimensional transesophageal echocardiography: implications for catheter-based left atrial appendage closure, Circ Cardiovasc Imaging 4(5) (2011) 514-23. 